\title{
BER Performance of OFDM-BPSK over Nakagami Fading Channels
}

\author{
Mukesh Kumar Mishra \\ Department of Electronics and \\ Communication Engineering \\ National Institute of \\ Technology Jalandhar \\ India
}

\author{
Neetu Sood \\ Department of Electronics and \\ Communication Engineering \\ National Institute of \\ Technology Jalandhar, \\ India
}

\author{
Ajay K Sharma \\ Department of Computer \\ Science Engineering \\ National institute of \\ Technology Jalandhar, \\ India
}

\begin{abstract}
In this paper, the Bit Error Rate (BER) performance of OFDM -BPSK system over flat Nakagami fading channels is analyzed. This model is versatile enough to represent fading. Here our approach is based on decomposition of Nakagami random variable into orthogonal random variables with Gaussian distribution envelopes. Finally simulations of OFDM signals are carried with Nakagami faded signal to understand the effect of channel fading and to obtain optimum value of $m, n$ and $\mathrm{q}$ based on BER and SNR.
\end{abstract}

\section{Keywords}

OFDM, fading distribution, Nakagami-m channel, Nakagami$\mathrm{n}$ channel, Nakagami-q channel, Rayleigh fading, Rician fading channel.

\section{INTRODUCTION}

Orthogonal frequency division multiplexing (OFDM) is an important wideband transmission technique for wireless communication systems. Compared with the other competing wideband transmission technology i.e. multicarrier code division multiple access an OFDM system can reduce or eliminate inter symbol interference (ISI) and is particularly suitable for transmission over fading channel requiring only a relatively simple equalizer at the receiver for a good performance. Nakagami distribution has been employed as another useful and important model for characterizing the fading channel, specially Nakagami-m fading which has fading figure from 0 to 2 .Another advantage of this channel is best fit for modeling urban multipath channels, could be better or more severe than Rayleigh fading channel.

Rayleigh and Rician fading channels have already been deployed and studied in depth for OFDM systems. In [1] and [2] OFDM signal on Rayleigh faded channel and Rician fading channels are studied in depth and BER expression is derived in frequency selective channels. In[3] The Nakagami distribution has been employed as another useful model for characterizing the amplitude of fading channels and both theoretical and the practical importance of the Nakagami-m, Nakagami-n and Nakagami-q channels have motivated intensive research into studying the performance of various communication systems operating in such channel. In [4] the purpose of this paper is to clarify quantitatively system performances of the MPSK signal transmitted through a slow and flat m-distributed fading channel. In[5] Alouini and Goldsmith used a MGF technique to study the error performance of different modulation techniques over Nakagami-m fading channel.[6] in this paper to characterize the Nakagami-m fading distribution fading channel claimed that the distribution of the frequency domain channel impulse response can be approximated by another Nakagami $m$ distribution with a new fading parameter different from the time domain fading parameter. In [7] modeled the OFDMBPSK system by presenting an approximate BER performance of OFDM system in frequency selective Nakagami-m channel using Characteristics Function approach and claimed that for Nakagami-m distribution, if we increase the value of $\mathrm{m}$ it is not necessary that the performance will always increases.

OFDM systems have gained an equivalent attention with flat fading environment In [8], present the method of channel estimation and Carrier frequency offset to design a channel is already presented in literature, so our motivation behind this paper is to study the performance of OFDM system using flat fading channel of all Nakagami distributions OFDM receiver in flat fading environment and to obtain the optimum value of $\mathrm{m}, \mathrm{n} \& \mathrm{q}$.

This paper is organized as follows: In section 2, OFDM system model is described. In section 3, the Nakagami fading channel is explained. In section 4, the mathematical model of channel is explained. In section 5 the analysis of simulated results of performance of OFDM system and finally section 6 , concludes the paper.

\section{MODEL DISCRIPTION}

A Complex base band OFDM signal with $\mathrm{N}$ subcarriers is expressed as [9]:

$$
\mathrm{s}(\mathrm{t})=\sum_{0}^{N-1} \mathrm{D}_{\mathrm{i}} \mathrm{e}^{\mathrm{j} / 2 \pi k f_{0} \mathrm{t}} \quad 0 \leq \mathrm{t} \leq \mathrm{T}
$$

For each OFDM symbol, the modulated data sequences are denoted by $D(0), D(1), \ldots, \ldots, D(N-1)$ Here, fo denote the sub-carriers spacing and is set to $f_{0}=\frac{1}{T}$ the condition of orthogonality. After IFFT, the time-domain OFDM signal can be expressed as [7]:

$S(n)=\frac{1}{N} \sum_{k=0}^{N-1} D_{i} e^{\frac{j 2 \pi k J_{0} n}{N}}$

After IFFT, the modulated signal is up-converted to carrier frequency $f_{c}$ and then the following signal is produced and transmitted through channel [9]:

$$
x(t)=\operatorname{Re}\left\{\sum_{k=0}^{N-1} D_{i} e^{j 2 \pi k\left(f_{0}+f_{c}\right) t}\right\} \quad 0 \leq t \leq T
$$

$\mathrm{x}(\mathrm{t})$ represents the final OFDM signal in which sub-carrier shall undergo a flat fading channel. 


\section{FADING DISTRIBUTION}

The following Nakagami distributions have been considered for our work:

\subsection{Nakagami-m Distribution}

Nakagami-m fading distribution has gained a lot of attention in the modeling of physical fading radio channels [10]. Nakagami-m is more flexible and it can model fading condition from worst to moderate. The reason behind taking this distribution is its good fit to empirical fading data. Due to free parameter it provides more flexibility. Nakagami-m fading distribution function is given by [3]:

$p_{\propto}(\propto)=\frac{2 m^{m} \propto^{2 m}-1}{\Gamma(m) \Omega^{m}} e^{-\frac{m x^{2}}{n}} \quad \propto \geq 0$

Where, $\Gamma($.$) is the Gamma function, \Omega=\overline{r^{2}}$ is the average power, $\mathrm{m}$ is fading parameter and $r$ is Nakagami distribution envelope. Since, Nakagami distribution encompasses Scattered, reflected and direct components of the original transmitted signal, it can be generated using the envelope of the both random signal processes $r_{\text {nlos }}(t)$ for non line- of- sight envelope i.e. Rayleigh and $r_{\text {los }}(t)$ for line-of-sight i.e. Rician as per the following expression.

$$
r(t)=\left|r_{\text {nlos }}(t)\right| e^{(1-m)}+\left|r_{\text {los }}(t)\right| \cdot\left(1-e^{(1-m)}\right)
$$

So, this value of $r(t)$ is used as envelope of Nakagami-m.

\subsection{Nakagami-q (Hoyt) Distribution}

The Nakagami-q distribution, also referred to as the Hoyt distribution is given by Nakagami [3, Eq. (52)] by;

$$
\begin{aligned}
& p_{\propto}(\propto)=\frac{\left(1+q^{2}\right) \propto x}{q \Omega} \exp \left(-\frac{\left(1+q^{2}\right) c^{2}}{4 q^{2} \Omega}\right) \\
& I_{0}\left(\frac{\left(1-q^{2}\right) \alpha^{2}}{4 q^{2} \Omega}\right) \propto \geq 0
\end{aligned}
$$

Where $\mathrm{I}_{0}$ (.) is zeroth order modified Bessel function of the first kind, and $\mathrm{q}$ is the Nakagami-q fading parameter which ranges from 0 to 1 .

\subsection{Nakagami-n (Rice) Distribution}

The Nakagami-n distribution is also known as the Rice distribution. It is often used to model propagation paths consisting of one strong direct LOS component and many random weaker components. Here the channel fading amplitude follows the distribution [3, Eq. (50)]

$$
\begin{gathered}
p_{\propto}(\propto)=\frac{2\left(1+n^{2}\right) e^{-n^{2}} \propto}{n} \exp \left(-\frac{\left(1+n^{2}\right) \propto c^{2}}{n}\right) \\
I_{0}\left(2 n \propto \sqrt{(} \frac{1+n^{2}}{\Omega}\right) \propto \geq 0
\end{gathered}
$$

Where $\mathrm{I}_{0}$ (.) is zeroth order modified Bessel function of the first kind, and $\mathrm{n}$ is the Nakagami-n fading parameter which ranges from 0 to $\infty$.

\section{CHANNEL MODEL}

In this paper, the sub-channel spacing $\left(f_{0}=1 / T\right)$ is chosen so that the produced parallel fading sub-channels have flat fading characteristics. In flat fading environment, the base-band signal at the input of receiver $y(t)$ is as described as follows [8]:

$y(t)=x(t) * r(t)+n(t)$

Where, $x(t)$ denotes the base-band transmitted signal, $r(t)$ is the Nakagami- $m$ channel envelope and $n(t)$ is the additive white Gaussian noise with zero mean

\section{RESULTS \& DISCUSSION}

To analyze the performance of OFDM-systems over Nakagami fading channel, we consider the Total number of sub-carriers 400, the IFFT /FFT length is chosen to be 1024 by using Guard interval of length 256 . In this section, we have presented the simulation results using MATLAB ${ }^{\mathrm{TM}}$ by implementing BPSK modulation formats for OFDM to get threshold value of fading parameter $\mathrm{m}, \mathrm{n}$ and $\mathrm{q}$. Figure 1 indicates the BER Versus SNR for OFDM-BPSK with different values of fading parameter $\mathrm{m}$. It is well known, that at $\mathrm{m}=1$, Nakagami-m fading corresponds to Rayleigh fading. So, the results for the same have been achieved through simulations. When value of $\mathrm{m}$ is increased, the BER starts reducing at $\mathrm{m}=1$.4.Further, if we increase $\mathrm{m}$, no reduction in BER has been reported rather it starts increasing. So threshold value of $\mathrm{m}$ is achieved to be 1.4 for Nakagami-m distribution, to estimate the fading channel. This interesting fact about Nakagami-m channel has also been reported by Zheng et al. [7] for frequency selective fading channel.

We have further analyzed OFDM system using Nakagami-n and Nakagami-q distribution and for Nakagami-n distribution threshold value is $\mathrm{n}=1.4$ and finally for Nakagami-q distribution threshold value is between .7 and .8 in our simulation this value is .75. These results are shown in Figure 2 and Figure-3. Results obtained for Nakagami-n and Nakagami-q OFDM-BPSK systems are similar in nature to that of Nakagami-m OFDM-BPSK system. In first three simulation we used guard type as zeroed signal to improve the result we use half zero, half cyclic guard type for Nakagami$\mathrm{m}$ fading channel and in simulation result gt1 represent Zeroed signal guard type and gt 3 represent half zero, half gt3 represent half zero, half cyclic guard type.

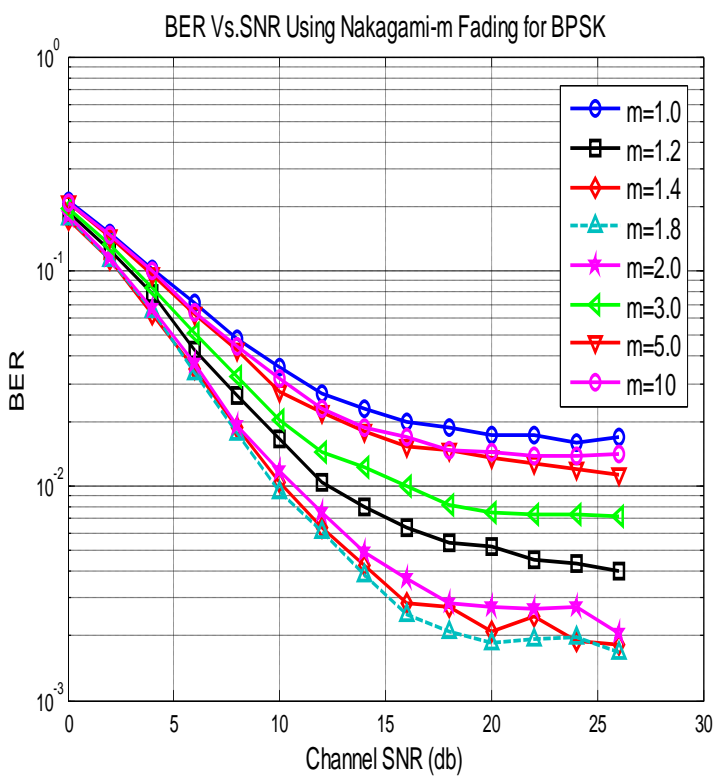

Fig.1 BER vs. SNR for OFDM-BPSK system over Nakagami-m Fading Channel 


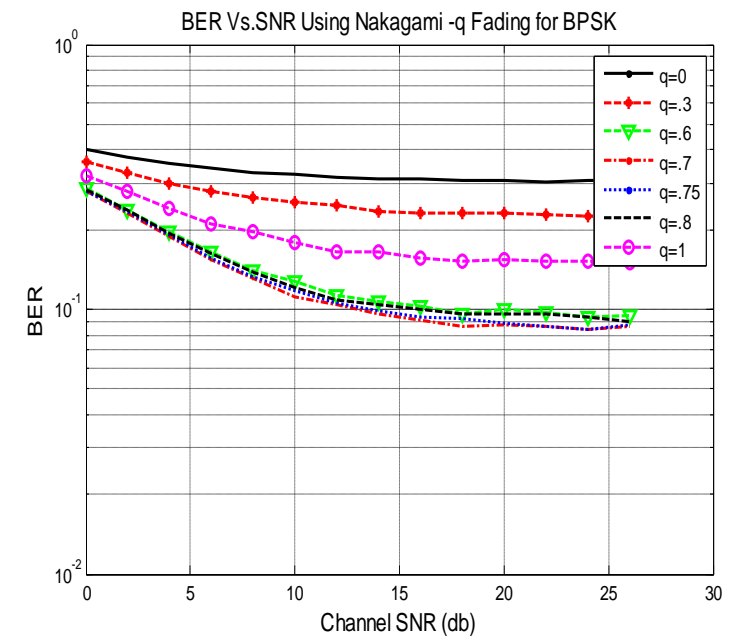

Fig. 3 BER vs. SNR for OFDM-BPSK system over Nakagami-q fading Channel BER Vs.SNR Using Nakagami-n Fading for BPSK

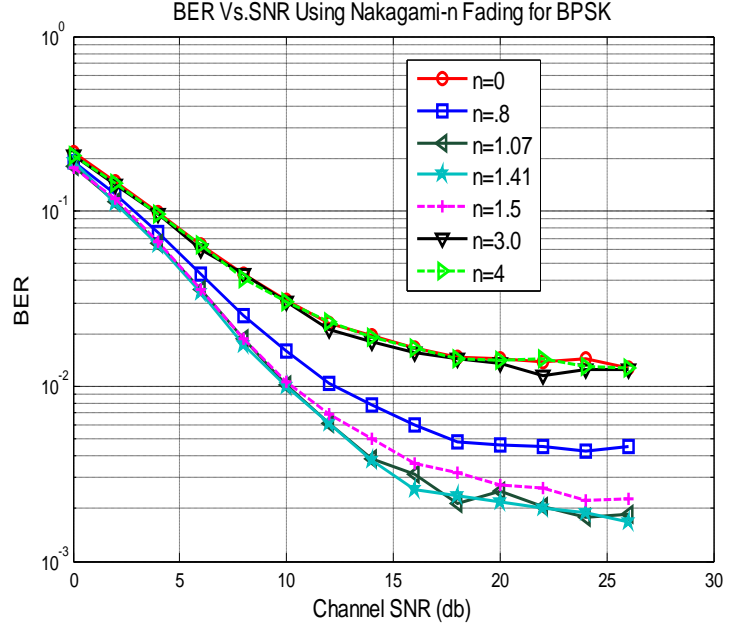

Fig. 3 BER vs. SNR for OFDM-BPSK system over Nakagami-n Fading Chanel

Finally, BER performance of OFDM system in Nakagami channel degrades if we increase $\mathrm{m}, \mathrm{n}$ and $\mathrm{q}$ beyond the certain threshold value.

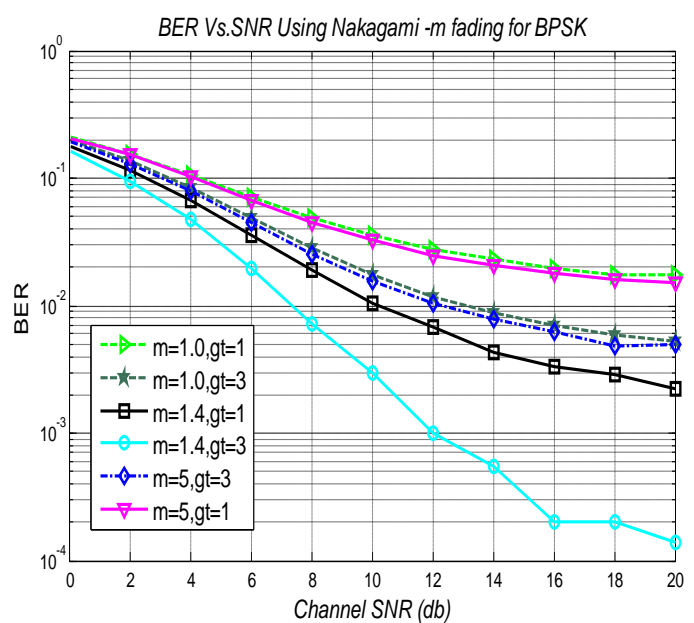

Fig. 4 BER vs. SNR for OFDM-BPSK system

\section{CONCLUSION}

In this paper, we have presented a method to evaluate the performance of OFDM system using BPSK with OFDM using Nakagami-m, Nakagami-n and Nakagami-q fading channel. Here approach is based on the decomposition of a Nakagami random variable into orthogonal random variables with Gaussian distributed envelopes. Results have been obtained for optimum value of $\mathrm{m}, \mathrm{n}$ and $\mathrm{q}$ which is useful for channel estimation of Nakagami distributions with flat fading environment for OFDM systems. The reported BER can be further reduced by using channel estimation or suitable diversity scheme.

\section{REFERENCES}

[1] Jin Goog Kim, Tae Joon and Jong Tae Lim, "Channel estimation for OFDM over Fast Rayleigh Fading Channels," Proceedings of world Academy of science and technology, vol. 21, pp. 455-458, Jan. 2007.

[2] Jun Lu, Thiang Tjhung, Fumiyuki Adachi and Cheng Li Huang, "BER performance of OFDM-MDPSK system in Frequency-Selective Rician Fading with Diversity Reception,"IEEE Trans. On Vehicular Tech., vol. 49, no. 4, pp. 1216-1225, July 2000.

[3] M. Nakagami, "The m-distribution-A general formula of intensity distribution of rapid fading," in Statistical Methods in Radio Wave Propagation, W. C. Hoffman, Ed. Elmsford, NY Pergamon, 1960.

[4] Yoshiya Miyagaki, N Moinaga," Error probability Characteristics for CPSK signal in Nakagami fading channel," IEEE Proc., Jan1978.

[5] M. Aloumini and A. J. Goldsmith, "A unified approach for calculating error rates of linearly modulated signals over fading channel", IEEE Trans. Commun., vol.47, no.9, pp. 1324-1334,Sep.1998

[6] Zhein gjiu Kang, Kung Yao, Flavio Lorenzelli, "Nakagami-m Fading Modeling in the Frequency Domain for OFDM system analysis," IEEE Communication letters, vol. 7, no.10, pp. 484-486, Oct.2003.

[7] Z. du, J. Cheng and N.C.Beaulieu, "Asymptotic BER performance of OFDM in Frequency selective Nakagamim Channels,"IEEE Conference on Vehicular Tech., vol. 1, pp. 612-615, Sept. 2004.

[8] Zhang yong Ma and Young-il- Kim, "A Novel OFDM receiver in Flat Fading Channel," IEEE Conference on advanced communication technology, ICACT, Vol. 2, pp. 1052-54, 2005.

[9] Neetu Sood, Ajay K Sharma, Moin Uddin, "BER Perfor mance of OFDM-BPSK and -QPSK over Nakagami-m Fading Channels", Proc. Of 2nd IEEE International Advance Computing Conference, IACC-2010, pp 88-90, Feb. 2010.

[10] Marvin K. Simon, Mohamed- Slim Alouini," Digital Communication over Fading Channels", John Willy \& Sons, 2000.

[11] J. G. Proakis, "Digital Communications", 3rd Ed. New York: McGraw-Hill, 1995. 